\title{
Maternal iron intake during pregnancy and birth outcomes: a cross-sectional study in Northwest China
}

\author{
Jiaomei Yang ${ }^{1}$, Yue Cheng ${ }^{2}$, Leilei Pei $^{1}$, Yufen Jiang ${ }^{1,3}$, Fangliang Lei ${ }^{1}$, Lingxia Zeng ${ }^{1}$, Quanli Wang ${ }^{1}$, \\ Qiang $\mathrm{Li}^{1}$, Yijun Kang ${ }^{1}$, Yuan Shen ${ }^{1}$, Shaonong Dang ${ }^{1 *}$ and Hong Yan ${ }^{1,4_{*}}$ \\ ${ }^{1}$ Department of Epidemiology and Health Statistics, School of Public Health, Xi'an Jiaotong University Health Science Center, \\ No. 76 Yanta West Road, Xi'an, Shaanxi, 710061, People's Republic of China \\ ${ }^{2}$ Department of Nutrition, School of Public Health, Xi'an Jiaotong University Health Science Center, Xi'an, Shaanxi, \\ 710061, People's Republic of China \\ ${ }^{3}$ Department of Obstetrics and Gynecology, Northwest Women's and Children's Hospital, Xi'an, Shaanxi, 710054, People's \\ Republic of China \\ ${ }^{4}$ Nutrition and Food Safety Engineering Research Center of Shaanxi Province, Xi'an, Shaanxi, 710061, People's Republic of China \\ (Submitted 5 November 2016 - Final revision received 21 February 2016 - Accepted 28 February 2017 - First published online 10 April 2017)
}

\section{Abstract}

Previous studies have yielded conflicting results on the associations of maternal Fe intake with birth outcomes. This study aimed to investigate the associations between maternal $\mathrm{Fe}$ intake (total $\mathrm{Fe}$ from diet and supplements, dietary total $\mathrm{Fe}$, haeme $\mathrm{Fe}$, non-haeme $\mathrm{Fe}$ and $\mathrm{Fe}$ supplements use) and adverse birth outcomes in Shaanxi Province of Northwest China. In all, 7375 women were recruited using a stratified multistage random sampling method at 0-12 months (median 3; 10th-90th percentile 0-7) after delivery. Diets were collected by a validated FFQ and maternal characteristics were obtained via a standard questionnaire. The highest tertile of haeme Fe intake compared with the lowest tertile was negatively associated with low birth weight (LBW) (OR 0.68; $95 \%$ CI 0.49, 0.94), small for gestational age (SGA) (OR 0.76; $95 \%$ CI $0.62,0.94$ ) and birth defects (OR 0.55; $95 \%$ CI 0.32, 0.89). Maternal haeme Fe intake was associated with a lower risk of intra-uterine growth retardation (IUGR) (medium tertile $v$. lowest tertile: OR 0.78; $95 \%$ CI 0.61, 0.95; highest tertile $v$. lowest tertile: OR 0.76; $95 \%$ CI 0.59, 0.93; $\left.P_{\text {trend }}=0.045\right)$. The OR of LBW associated with Fe supplements use were as follows: during pregnancy: 0.72 (95\% CI 0.50, 0.95$)$; in the second trimester: 0.67 (95\% CI 0.42, 0.98); in the third trimester: 0.47 (95\% CI 0.24, 0.93). We observed no associations of total Fe, dietary total Fe or non-haeme Fe intake with birth outcomes. The results suggest that maternal haeme Fe intake is associated with a reduced risk of LBW, SGA, IUGR and birth defects, and Fe supplements use during pregnancy reduces LBW risk.

Key words: Dietary iron: Iron supplements: Pregnancy: Birth weight: Gestational age

Fe deficiency is the most common nutritional deficiency among pregnant women in both the developed and the developing world $^{(1)}$. During pregnancy Fe requirements considerably increase because of the expansion of maternal red cell mass and the growth of fetal-placental unit ${ }^{(1)}$. Evidence exists that prenatal Fe use improves birth weight ${ }^{(2)}$, gestational duration ${ }^{(3)}$ and child intellectual development ${ }^{(4)}$, though some studies have not confirmed the associations ${ }^{(5,6)}$. Animal studies suggested that maternal Fe deficiency in pregnancy affected blood pressure and cardiovascular development in the offspring ${ }^{(7,8)}$.

The main source of body Fe stores is from diets. Dietary Fe exists in two forms: haeme and non-haeme Fe. Haeme Fe comes mainly from animals and is better absorbed than non-haeme Fe that comes mainly from plants ${ }^{(9)}$. Studies suggested that haeme and non-haeme Fe may be differently associated with health outcomes $^{(10,11)}$. Dietary Fe inadequacy during pregnancy is prevalent worldwide, especially in the developing countries ${ }^{(12)}$. To meet the increased Fe requirements, Fe supplementation has been routinely recommended during pregnancy in the USA and Canada. However, because of the inconsistent effects of prenatal Fe use on birth outcomes and possible gastrointestinal side effects, the routine Fe supplements use during pregnancy is not recommended in most countries.

The relationship between dietary Fe intake and adverse birth outcomes such as low birth weight (LBW), preterm birth (PTB), small for gestational age (SGA) and birth defects has been explored in several studies ${ }^{(13-18)}$. However, the results were conflicting. Moreover, few studies considered the potential different effects of different sources of Fe (dietary or supplemental) and types of Fe (haeme or non-haeme) on birth outcomes ${ }^{(16)}$. The evidence for the effects of vitamin C, smoking, alcohol drinking and anaemia on the associations is also limited ${ }^{(15,16)}$. To our knowledge, the

Abbreviations: IUGR, intra-uterine growth retardation; LBW, low birth weight; PTB, preterm birth; RNI, recommended nutrient intake; SGA, small for gestational age.

* Corresponding authors: H. Yan, fax +86 29 82655104, email xjtu_yh2014@163.com; S. Dang, fax +86 29 82655104, email tjdshn@mail.xjtu.edu.cn 
associations between dietary and supplemental Fe intakes during pregnancy and birth outcomes have not been reported in China. China has been reported to undergo a nutrition transition during the past few decades, with animal-source foods intakes increasing markedly ${ }^{(19)}$. Pregnant women in Northwest China reported dietary Fe intake close to the recommended nutrient intake (RNI); however, they may have poor Fe absorption because of the high proportion of plant foods contributing to $\mathrm{Fe}$ intake and the low intake of vitamin $\mathrm{C}^{(20)}$. In China, Fe supplements use during pregnancy is still not common. The present study conducted in Shaanxi Province of Northwest China aimed to examine the associations of maternal $\mathrm{Fe}$ intake (total $\mathrm{Fe}$ from diet and supplements, dietary total Fe, haeme Fe, non-haeme Fe and Fe supplements use) during pregnancy with birth outcomes (LBW, PTB, SGA, intra-uterine growth retardation (IUGR) and birth defects), and to investigate the potential effect modifications by vitamin $\mathrm{C}$, passive smoking, alcohol drinking and anaemia.

\section{Methods}

\section{Study design and participants}

A population-based cross-sectional survey with the purpose of investigating the risk factors for birth outcomes and maternal nutritional status during pregnancy was conducted in Shaanxi, China between August and November 2013. This area can be divided into three regions: northern, southern and central Shaanxi, with natural resources, history, culture and lifestyle greatly differing among them. A total of 30027 women who were pregnant during 2010-2013 were recruited in the survey using a stratified multistage random sampling method. According to the proportion of rural to urban residents, population size and fertility rate in Shaanxi, China, twenty counties and ten districts were randomly sampled. In each sampled county, six villages each from six townships were randomly selected; in each sampled district, six communities each from three streets were randomly selected. In all, thirty and sixty participants were randomly selected in each sampled village and community, respectively. Among the participants, 7750 women who were pregnant during 2012-2013 and had infants $<12$ months old were further interviewed to report their diets during pregnancy. Women who reported a multiple gestation or an implausible total energy intake (>20920 or $<2092 \mathrm{~kJ} / \mathrm{d}$ ) were excluded from the present study. The final sample included a total of 7375 eligible mothers, and the median month after delivery when mothers were interviewed was 3 (10th-90th percentiles 0-7). The flow diagram of sampling strategy with exclusion criteria is displayed in Fig. 1.

\section{Ethics}

This study was conducted in accordance with the Helsinki Declaration, and all procedures involving human subjects were approved by the Xi'an Jiaotong University Health Science Center. Written informed consent was obtained from all participants.

\section{Assessment of nutrient intake}

Dietary intakes of mothers during the whole pregnancy were measured by a 107 -item semi-quantitative FFQ at 0-12 months (median 3; 10th-90th percentiles 0-7) after delivery. Maternal

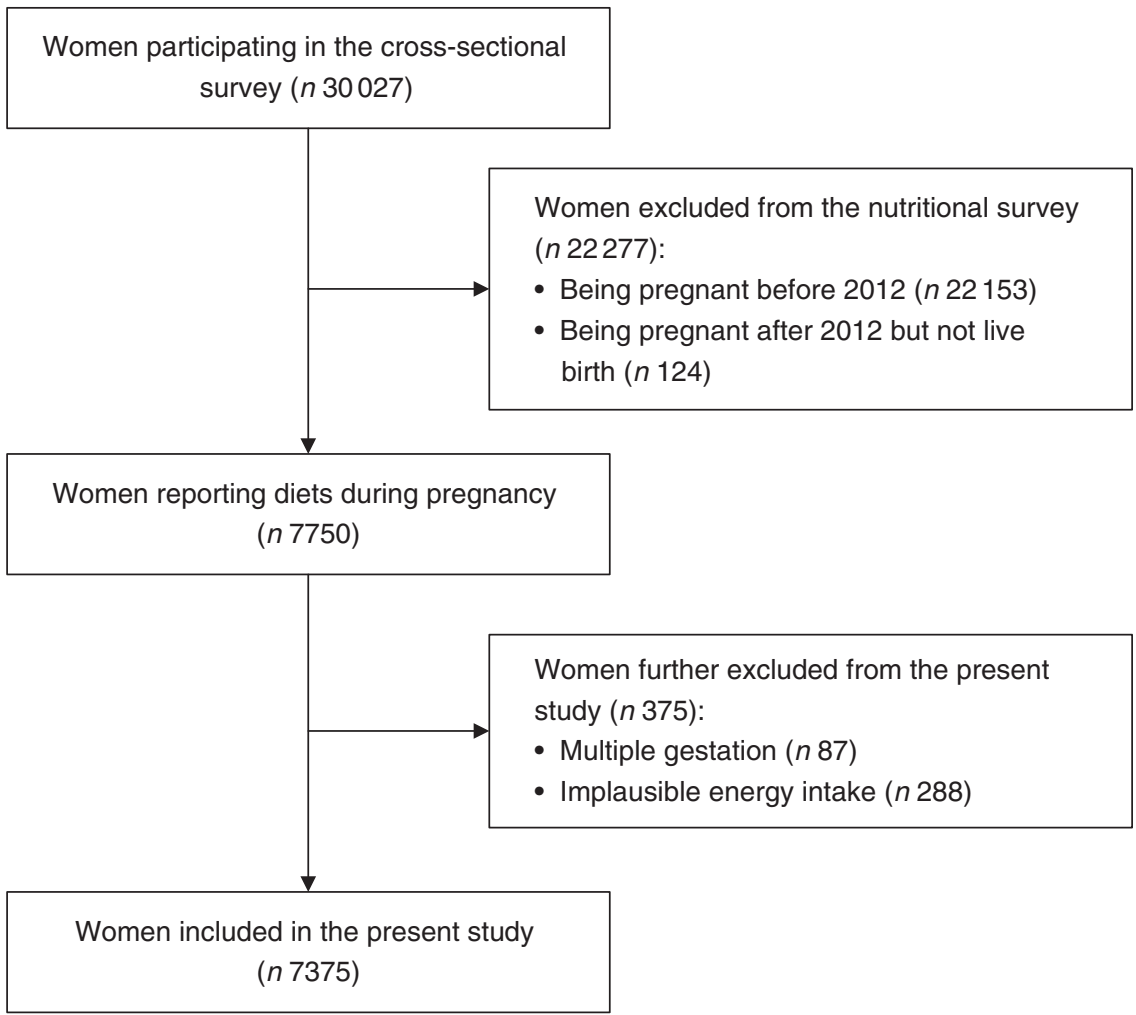

Fig. 1. Flow diagram of sampling strategy with exclusion criteria. 
dietary patterns tended to change little from early to late pregnancy ${ }^{(21)}$. Diet assessment during the whole pregnancy at one time was reasonable, convenient and economical for large epidemiological studies ${ }^{(22)}$, especially for our present largescale survey with multiple dietary exposures and outcomes ${ }^{(20)}$. The FFQ was established on the basis of the previously validated FFQ used for pregnant women in Shaanxi, China ${ }^{(23)}$. Pearson's correlation coefficient for Fe between the FFQ and the average of six $24-\mathrm{h}$ recalls was 0.65 , with a range of $0.53-0.70$ for other nutrients ${ }^{(23)}$. The frequency scales of five food items (animal oils, vegetable oils, salt, sugar and sauce) were open-ended, and were recorded as kilograms per month and the number of people regularly consuming them. Regarding the other 102 food items, women were asked to recall consumption frequency from the eight predefined categories ranging from never to two or more times per day and identify their portion sizes according to food portion images ${ }^{(20)}$. Daily intakes of dietary total Fe, haeme Fe (mainly derived from animal meat sources), non-haeme Fe (derived from the difference between dietary total $\mathrm{Fe}$ and haeme $\mathrm{Fe}$ ) and other nutrients were transformed using the China Food Composition Tables ${ }^{(24,25)}$. Participants were also asked to report the type/ brand, lasting day and the amount of all dietary supplements they used during each trimester of pregnancy. Daily supplemental Fe intake was calculated by multiplying the Fe content of each reported supplement and the number of supplement tablets taken daily. Total Fe intake was derived from the sum of dietary $\mathrm{Fe}$ and supplemental $\mathrm{Fe}$ intakes. The RNI of Fe for Chinese pregnant women during the first, second and third trimesters were 20,24 and $29 \mathrm{mg} / \mathrm{d}$, respectively ${ }^{(26)}$.

\section{Ascertainment of birth outcomes}

Neonatal information including birth date, sex, birth weight and gestational age was obtained by reviewing birth certificates. Birth weight was measured with a baby scale with precision to the nearest $10 \mathrm{~g}$. Gestational age at delivery was calculated according to the last menstrual period, and was confirmed by ultrasound scans. Birth defects information was collected with the pre-code structured questionnaire according to the International Classification of Diseases, 10th revision. Medical records including clinical diagnosis, physical examination, ultrasound imaging report and medical history were referred to ascertain birth outcomes. Birth outcomes were defined as follows: (1) LBW, birth weight <2500 g; (2) PTB, delivery $<37$ completed gestational week; (3) SGA, birth weight $<10$ th percentile of the gestational age-sex specific Chinese reference for fetal growth ${ }^{(27)}$; (4) IUGR, birth weight $<3$ rd percentile of the gestational age-sex specific Chinese reference for fetal growth $^{(27)}$.

\section{Assessment of socio-demographic and health-related characteristics}

The general information of the participants during pregnancy was gathered face to face by well-trained interviewers via a standard questionnaire. The study information was classified as the following: (1) Socio-demographic characteristics: geographic area (northern, southern or central Shaanxi); residence (rural or urban); childbearing age ( $<25,25-29$ or $\geq 30$ years); maternal education (primary school or below, junior high school or senior high school or above); maternal occupation (farmer or working outside); nulliparity (yes or no); (2) health-related characteristics: passive smoking (yes or no); alcohol drinking (yes or no); frequency of antenatal care visits $(<6$ or $\geq 6$ ); folate supplements use (yes or no); anaemia (yes or no); medication use (yes or no). Passive smoking was defined as being exposed to another person's tobacco smoke for $\geq 15 \mathrm{~min} / \mathrm{d}$. Alcohol drinking included a wide range of alcoholic beverages (liquor, wine and beer) consumed during pregnancy. Anaemia during pregnancy was diagnosed using the criteria of hb concentration $<110 \mathrm{~g} / \mathrm{l}$.

\section{Statistical analysis}

The study population characteristics and energy-adjusted nutrient intakes by residual $\operatorname{method}^{(28)}$ were described according to tertiles of dietary total $\mathrm{Fe}$ and haeme $\mathrm{Fe}$ intakes. Categorical variables were expressed as percentages and continuous variables as means. Univariable comparisons were made using $\chi^{2}$ test for categorical variables and ANOVA for continuous variables. Household wealth index was established by principal component analysis according to the variables of family economic level (housing condition, vehicle type, income source, and type and number of household appliance), and this index was divided into thirds as an indicator for the poor, medium and rich households ${ }^{(29)}$. To avoid multicollinearity of nutrients in regression analysis, principal component analysis was used according to the intakes of potential nutrients (protein, fat, carbohydrate, vitamin A, thiamin, riboflavin, folate, vitamin C, vitamin $\mathrm{E}, \mathrm{Ca}, \mathrm{Zn}$ and Se). The number of components extracted was based on eigenvalues $>1$, the scree plot and interpretability $^{(30)}$. The first component that explained $68.7 \%$ of the total variance was finally extracted, and the factor loadings of protein, $\mathrm{Zn}$, riboflavin, thiamin, $\mathrm{Ca}$ and Se were above $0 \cdot 80$.

In our study, the survey data collected from the stratified multistage random sampling design had a hierarchical structure in which lower levels were nested within higher levels. Considering this, multilevel models which were explicitly designed to analyse hierarchically structured data were applied. We first constructed four-level empty models representing county (district)-township (community)-village (street)-individual and observed non-significant within-group variations (all $P>0.05$ ) and low intra-class correlations (all lower than 0.001) in the village (street) level. Therefore, we adopted simplified multilevel models with a random intercept at the county (district) and the township (community) levels. Multilevel logistic regression models were used to estimate OR and 95\% CI for adverse birth outcomes (LBW, PTB, SGA, IUGR and birth defects) associated with maternal $\mathrm{Fe}$ intake and $\mathrm{Fe}$ supplements use, and multilevel linear regression models were used to evaluate the effect of every $1 \mathrm{mg} / \mathrm{d}$ increase in maternal haeme Fe intake on birth weight. The intake of total $\mathrm{Fe}$, dietary $\mathrm{Fe}$, haeme $\mathrm{Fe}$ and non-haeme Fe was stratified by tertile to avoid the possible influence of extreme values, whereas the Fe supplements use was categorised by 
trimester because of the low proportion of pregnant women taking Fe supplements in China and the possible different effects of $\mathrm{Fe}$ supplements use on birth outcomes during different trimesters reported before ${ }^{(16)}$. Four adjusted models were established: (1) model 1 adjusted for energy; (2) model 2 adjusted for energy and socio-demographic characteristics, including geographic area, residence, childbearing age, education, occupation, household wealth index and parity; (3) model 3 adjusted for all variables in model 2 plus health-related characteristics, including passive smoking, alcohol drinking, antenatal care visit frequency, folate supplements use, anaemia and medication use; (4) model 4 adjusted for all variables in model 3 plus principal component score based on the nutrient intakes. Models were additionally adjusted for Fe supplements use to examine the effect of dietary total $\mathrm{Fe}$, adjusted for $\mathrm{Fe}$ supplements use and non-haeme Fe to examine the effect of haeme $\mathrm{Fe}$, adjusted for $\mathrm{Fe}$ supplements use and haeme $\mathrm{Fe}$ to examine the effect of non-haeme $\mathrm{Fe}$, and additionally adjusted for dietary total Fe to examine the effect of Fe supplements use. To test for a linear trend, we used the median for each tertile of iron intake as a continuous variable. We further conducted stratified analyses and evaluated interaction terms to explore the possible modifiable effects of vitamin C intake (the RNI of $\geq$ or $<115 \mathrm{mg} / \mathrm{d}$ for Chinese pregnant women), passive smoking (yes or no), alcohol drinking (yes or no) and anaemia (yes or no) on the associations.

Statistical analyses were performed using STATA software (version 12.0; StataCorp LP). A two-tailed $P<0.05$ was regarded as statistically significant.

\section{Results}

\section{Status of iron intake}

The status of Fe intake among pregnant women in Shaanxi, China is present in Table 1. Mean total Fe intake from diet and supplements was $29 \cdot 8$ (SD 12.7 ) $\mathrm{mg} / \mathrm{d}$, and the proportions of participants reporting total $\mathrm{Fe}$ intake below the RNI of 20, 24 and $29 \mathrm{mg} / \mathrm{d}$ were $27.9,38.1$ and $51.1 \%$, respectively. Mean dietary total Fe intake was 28.2 (sD 11.3) $\mathrm{mg} / \mathrm{d}$, and the proportions of women who had dietary Fe intake below the RNI of 20,24 and $29 \mathrm{mg} / \mathrm{d}$ were $30.2,40.9$ and $53.8 \%$, respectively. Mean haeme Fe intake was 2.6 (SD 2.3 ) $\mathrm{mg} / \mathrm{d}$, and the main

Table 1. Daily iron intake from different sources among pregnant women in Shaanxi Province, Northwest China

(Mean values and standard deviations)

\begin{tabular}{lrr}
\hline & Mean & \multicolumn{1}{c}{ SD } \\
\hline Total Fe intake from diet and supplements $(\mathrm{mg} / \mathrm{d})$ & 29.8 & 12.7 \\
Dietary total Fe intake $(\mathrm{mg} / \mathrm{d})$ & 28.2 & 11.3 \\
Haeme $\mathrm{Fe}$ intake $(\mathrm{mg} / \mathrm{d})$ & 2.6 & 2.3 \\
Fe intake from red meat $(\mathrm{mg} / \mathrm{d})^{\star}$ & 2.0 & 1.6 \\
Fe intake from white meat $(\mathrm{mg} / \mathrm{d}) \dagger$ & 0.6 & 0.5 \\
Non-haeme Fe intake $(\mathrm{mg} / \mathrm{d})$ & 25.6 & 11.3 \\
$\mathrm{Fe}$ intake from cereals and tubers $(\mathrm{mg} / \mathrm{d})$ & 15.9 & 11.9 \\
$\mathrm{Fe}$ intake from vegetables and fruits $(\mathrm{mg} / \mathrm{d})$ & 5.5 & 3.3 \\
Fe intake from legumes and nuts $(\mathrm{mg} / \mathrm{d})$ & 3.0 & 2.7 \\
\hline
\end{tabular}

* Red meat included pork, beef, mutton and their products.

† White meat included poultry, fish, shrimp, shellfish and their products. contributor to haeme Fe intake was red meat (76.9\%). Mean non-haeme Fe intake was 25.6 (sD 11.3 ) $\mathrm{mg} / \mathrm{d}$, with cereals and tubers contributing the most to non-haeme Fe intake (62.1\%). $19.8 \%$ of participants reported taking $\mathrm{Fe}$ supplements during pregnancy, and women with Fe supplements use during the first, second and third trimesters accounted for 6.9, $12 \cdot 1$ and $8.2 \%$ of the sample, respectively. Only $5.4 \%$ of women reported taking Fe-only supplements during pregnancy, with those taking Fe-only supplements during the three gestational periods accounting for $2 \cdot 1,2.9$ and $2.5 \%$ of the sample, respectively.

\section{Characteristics of the participants}

The general characteristics, energy-adjusted nutrient intakes and pregnancy outcomes among the participants according to tertiles of dietary total $\mathrm{Fe}$ and haeme Fe intakes are displayed in Table 2. Pregnant women with higher dietary total Fe and haeme Fe intakes tended to be in central Shaanxi and southern Shaanxi, respectively. Participants with higher dietary total $\mathrm{Fe}$ and haeme $\mathrm{Fe}$ intakes were more likely to live in urban areas, be aged 25-29 years at delivery, be better educated, work outside, be wealthier, have more antenatal care visits and take supplements of folate and Fe. Women with higher dietary total Fe intake were less likely to drink during pregnancy, and women with higher haeme Fe intake were less likely to be exposed to secondhand smoke. Pregnant women consuming more haeme Fe also tended to be at their first delivery.

Significant differences existed in all selected energy-adjusted nutrient intakes among tertiles of dietary total $\mathrm{Fe}$ and haeme Fe intakes. Dietary total Fe intake was positively associated with most nutrients intake, and negatively associated with fat, vitamin $\mathrm{E}$ and Se intake. Haeme $\mathrm{Fe}$ intake was positively associated with most nutrients intake, and negatively associated with dietary total $\mathrm{Fe}$, non-haeme $\mathrm{Fe}$, carbohydrate and vitamin E intake.

Pregnancy outcomes of the participants were as follows: (1) birth weight, 3264 (sD 440) g; (2) gestational age, 39.6 (SD 1.3) weeks; (3) LBW, $3.3 \%$; (4) PTB, $3.1 \%$; (5) SGA, $12.8 \%$; (6) IUGR, $5.8 \%$; (7) birth defects, $1.9 \%$. The results of univariable comparisons suggested significant differences in birth weight, LBW, SGA and IUGR among tertiles of haeme Fe intake.

\section{Associations between total iron intake from diet and supplements and birth outcomes}

Total Fe intake from diet and supplements was not associated with LBW, PTB, SGA, IUGR or birth defects and the tests for trend were not statistically significant in all models (online Supplementary Table S1).

\section{Associations between dietary iron intakes and birth outcomes}

There was no relationship between dietary total Fe intake and adverse birth outcomes (online Supplementary Table S1). The associations of dietary haeme Fe and non-haeme Fe intakes 
Table 2. Baseline characteristics of participants by tertiles (T) of dietary total iron and haeme iron intakes among pregnant women in Shaanxi Province, Northwest China

(Mean values and percentages)

\begin{tabular}{|c|c|c|c|c|c|c|c|c|}
\hline & \multicolumn{4}{|c|}{ Dietary total Fe intake } & \multicolumn{4}{|c|}{ Haeme Fe intake } \\
\hline & Tertile 1 & Tertile 2 & Tertile 3 & $P^{\star}$ & Tertile 1 & Tertile 2 & Tertile 3 & $P^{*}$ \\
\hline \multicolumn{9}{|l|}{ General characteristics } \\
\hline Geographic area (\%) & & & & $<0.001$ & & & & $<0.001$ \\
\hline Northern Shaanxi & $22 \cdot 0$ & $17 \cdot 1$ & $16 \cdot 8$ & & 22.9 & $19 \cdot 3$ & $13 \cdot 8$ & \\
\hline Southern Shaanxi & $38 \cdot 2$ & $29 \cdot 6$ & $10 \cdot 2$ & & 24.9 & $29 \cdot 2$ & $35 \cdot 0$ & \\
\hline Central Shaanxi & $39 \cdot 8$ & $53 \cdot 3$ & 64.0 & & $52 \cdot 3$ & 51.5 & $51 \cdot 2$ & \\
\hline Rural residence (\%) & $80 \cdot 1$ & 74.7 & $73 \cdot 4$ & $<0.001$ & $87 \cdot 4$ & $76 \cdot 4$ & $64 \cdot 3$ & $<0.001$ \\
\hline Childbearing age (years) (\%) & & & & $<0.001$ & & & & 0.043 \\
\hline$<25$ & $45 \cdot 9$ & $41 \cdot 0$ & $37 \cdot 5$ & & 42.9 & $41 \cdot 5$ & $40 \cdot 1$ & \\
\hline $25-29$ & $34 \cdot 8$ & $37 \cdot 2$ & $41 \cdot 3$ & & $35 \cdot 7$ & $37 \cdot 5$ & $40 \cdot 0$ & \\
\hline$\geq 30$ & $19 \cdot 3$ & $21 \cdot 8$ & $21 \cdot 3$ & & 21.4 & $21 \cdot 0$ & $19 \cdot 9$ & \\
\hline Maternal education (\%) & & & & $<0.001$ & & & & $<0.001$ \\
\hline Primary school or below & $11 \cdot 4$ & $8 \cdot 3$ & $8 \cdot 0$ & & $14 \cdot 1$ & $7 \cdot 9$ & $5 \cdot 8$ & \\
\hline Junior high school & $56 \cdot 7$ & $53 \cdot 3$ & $53 \cdot 5$ & & $59 \cdot 2$ & 54.9 & 53.5 & \\
\hline Senior high school or above & 31.9 & $38 \cdot 4$ & $37 \cdot 3$ & & $26 \cdot 7$ & $37 \cdot 2$ & $37 \cdot 3$ & \\
\hline Farmer $(\%)$ & $75 \cdot 3$ & $71 \cdot 3$ & $67 \cdot 9$ & $<0.001$ & $78 \cdot 2$ & 71.7 & $64 \cdot 6$ & $<0.001$ \\
\hline Household wealth index (\%) & & & & 0.039 & & & & $<0.001$ \\
\hline Poor & 30.4 & $31 \cdot 7$ & $29 \cdot 5$ & & $31 \cdot 7$ & $33 \cdot 0$ & $26 \cdot 9$ & \\
\hline Medium & $38 \cdot 7$ & $36 \cdot 3$ & $36 \cdot 1$ & & 40.9 & $36 \cdot 1$ & $34 \cdot 1$ & \\
\hline Rich & 30.9 & $32 \cdot 0$ & 34.4 & & $27 \cdot 4$ & $30 \cdot 9$ & $39 \cdot 0$ & \\
\hline Nulliparity (\%) & $59 \cdot 6$ & $59 \cdot 4$ & $60 \cdot 1$ & 0.882 & $53 \cdot 6$ & $59 \cdot 8$ & $65 \cdot 7$ & $<0.001$ \\
\hline Passive smoking (\%) & $22 \cdot 2$ & $21 \cdot 5$ & $19 \cdot 9$ & 0.140 & $23 \cdot 3$ & $20 \cdot 9$ & $19 \cdot 4$ & 0.004 \\
\hline Alcohol drinking $(\%)$ & 1.8 & $1 \cdot 1$ & $1 \cdot 0$ & 0.020 & 0.9 & 1.4 & 1.6 & 0.116 \\
\hline More than six antenatal check visits (\%) & $52 \cdot 0$ & $56 \cdot 2$ & $56 \cdot 6$ & 0.002 & $44 \cdot 6$ & $55 \cdot 2$ & $65 \cdot 1$ & $<0.001$ \\
\hline Folate supplements use (\%) & 66.4 & $69 \cdot 2$ & $71 \cdot 3$ & 0.001 & $65 \cdot 2$ & $70 \cdot 3$ & 71.5 & $<0.001$ \\
\hline Fe supplements use (\%) & $17 \cdot 0$ & $19 \cdot 9$ & 22.5 & $<0.001$ & $15 \cdot 4$ & $20 \cdot 1$ & 23.9 & $<0.001$ \\
\hline Anaemia (\%) & $17 \cdot 5$ & $17 \cdot 9$ & $19 \cdot 9$ & 0.072 & $18 \cdot 9$ & $18 \cdot 9$ & $17 \cdot 5$ & 0.366 \\
\hline Medication use (\%) & $17 \cdot 7$ & $19 \cdot 7$ & $19 \cdot 8$ & 0.122 & $19 \cdot 3$ & $19 \cdot 0$ & $19 \cdot 1$ & 0.590 \\
\hline \multicolumn{9}{|l|}{ Energy-adjusted nutrient intakes } \\
\hline Energy $(\mathrm{kJ} / \mathrm{d})$ & 6753 & 9134 & 11268 & $<0.001$ & 7489 & 8699 & 10962 & $<0.001$ \\
\hline $\mathrm{Fe}(\mathrm{mg} / \mathrm{d})$ & 20 & 26 & 38 & $<0.001$ & 29 & 28 & 27 & 0.027 \\
\hline Haeme Fe (mg/d) & 2.5 & 2.7 & $2 \cdot 8$ & $<0.001$ & 1.9 & $2 \cdot 0$ & $4 \cdot 1$ & 0.027 \\
\hline Non-haeme Fe (mg/d) & 17 & 24 & 36 & $<0.001$ & 27 & 26 & 24 & 0.027 \\
\hline Protein $(\mathrm{g} / \mathrm{d})$ & 65 & 67 & 68 & $<0.001$ & 60 & 61 & 69 & $<0.001$ \\
\hline Fat $(g / d)$ & 90 & 86 & 81 & $<0.001$ & 77 & 80 & 90 & $<0.001$ \\
\hline Carbohydrate $(\mathrm{g} / \mathrm{d})$ & 300 & 311 & 323 & $<0.001$ & 314 & 306 & 274 & $<0.001$ \\
\hline Vitamin A ( $\mu \mathrm{g}$ retinol equivalent/d) & 306 & 441 & 512 & $<0.001$ & 279 & 328 & 752 & $<0.001$ \\
\hline Thiamin $(\mathrm{mg} / \mathrm{d})$ & 0.6 & 0.7 & 0.7 & 0.007 & 0.6 & 0.6 & 0.7 & $<0.001$ \\
\hline Riboflavin (mg/d) & 0.6 & 0.7 & 0.7 & $<0.001$ & 0.6 & 0.6 & 0.8 & $<0.001$ \\
\hline Folate $(\mu \mathrm{g} / \mathrm{d})$ & 278 & 289 & 318 & $<0.001$ & 258 & 264 & 310 & $<0.001$ \\
\hline Vitamin C (mg/d) & 58 & 60 & 63 & $<0.001$ & 56 & 57 & 60 & $<0.001$ \\
\hline Vitamin $E(\mathrm{mg} / \mathrm{d})$ & 41 & 40 & 39 & 0.019 & 41 & 40 & 39 & 0.028 \\
\hline $\mathrm{Ca}(\mathrm{mg} / \mathrm{d})$ & 549 & 566 & 586 & $<0.001$ & 520 & 533 & 556 & $<0.001$ \\
\hline $\mathrm{Zn}(\mathrm{mg} / \mathrm{d})$ & $6 \cdot 1$ & $6 \cdot 2$ & 6.5 & $<0.001$ & $5 \cdot 3$ & 5.7 & $6 \cdot 8$ & $<0.001$ \\
\hline Se $(\mathrm{mg} / \mathrm{d})$ & 34 & 33 & 32 & 0.001 & 27 & 29 & 38 & $<0.001$ \\
\hline \multicolumn{9}{|l|}{ Pregnancy outcomes } \\
\hline Birth weight $(\mathrm{g})$ & 3257 & 3266 & 3267 & 0.396 & 3231 & 3267 & 3294 & $<0.001$ \\
\hline Gestational age (weeks) & 39.5 & 39.6 & 39.6 & 0.157 & 39.5 & 39.6 & 39.6 & 0.233 \\
\hline Low birth weight (\%) & 3.6 & 3.4 & $3 \cdot 1$ & 0.377 & $4 \cdot 3$ & $2 \cdot 8$ & $2 \cdot 8$ & 0.004 \\
\hline Preterm birth (\%) & 3.7 & $3 \cdot 0$ & 2.5 & 0.052 & 3.6 & 2.9 & $2 \cdot 8$ & 0.236 \\
\hline Small for gestational age (\%) & $13 \cdot 0$ & $12 \cdot 8$ & $12 \cdot 6$ & 0.261 & $15 \cdot 2$ & $12 \cdot 7$ & $10 \cdot 5$ & $<0.001$ \\
\hline Intra-uterine growth retardation (\%) & 6.5 & $5 \cdot 6$ & 5.4 & 0.199 & $7 \cdot 6$ & $5 \cdot 3$ & 4.5 & $<0.001$ \\
\hline Birth defects $(\%)$ & $2 \cdot 2$ & 1.9 & $1 \cdot 8$ & 0.323 & 2.4 & 1.9 & 1.6 & 0.117 \\
\hline
\end{tabular}

* $P$ values for the differences among groups were derived from $X^{2}$ tests for categorical variables and ANOVA for continuous variables.

with birth outcomes are shown in Table 3. No associations of non-haeme Fe intake with LBW, SGA, IUGR or birth defects were observed. The highest tertiles of non-haeme Fe intakes compared with the lowest tertiles were significantly and negatively associated with PTB risk in model 1 (OR 0.65; $95 \%$ CI $0.43,0.97$ ) and in model 2 (OR 0.66; $95 \%$ CI 0.44, 0.98), and the tests for trend were significant in model $1\left(P_{\text {trend }}=0.039\right)$ and in model $2\left(P_{\text {trend }}=0 \cdot 047\right)$. However, the associations attenuated and were not significant after further adjustment for healthrelated factors in model 3 and further adjustment for healthrelated plus dietary factors in model 4 , and the tests for trend were not statistically significant in these two models $\left(P_{\text {trend }}>0 \cdot 05\right)$.

Participants in the highest tertile of $>2.65 \mathrm{mg} / \mathrm{d}$ haeme Fe intake were less likely to have a LBW baby (OR 0.68; $95 \%$ CI 0.49, 0.94) and a SGA baby (OR 0.76; $95 \%$ CI 0.62, 0.94) compared with those 
Table 3. Birth outcomes associated with tertiles $(\mathrm{T})$ of dietary haeme iron and non-haeme iron intakes during pregnancy in Shaanxi Province, Northwest China*

(Odds ratios and $95 \%$ confidence intervals)

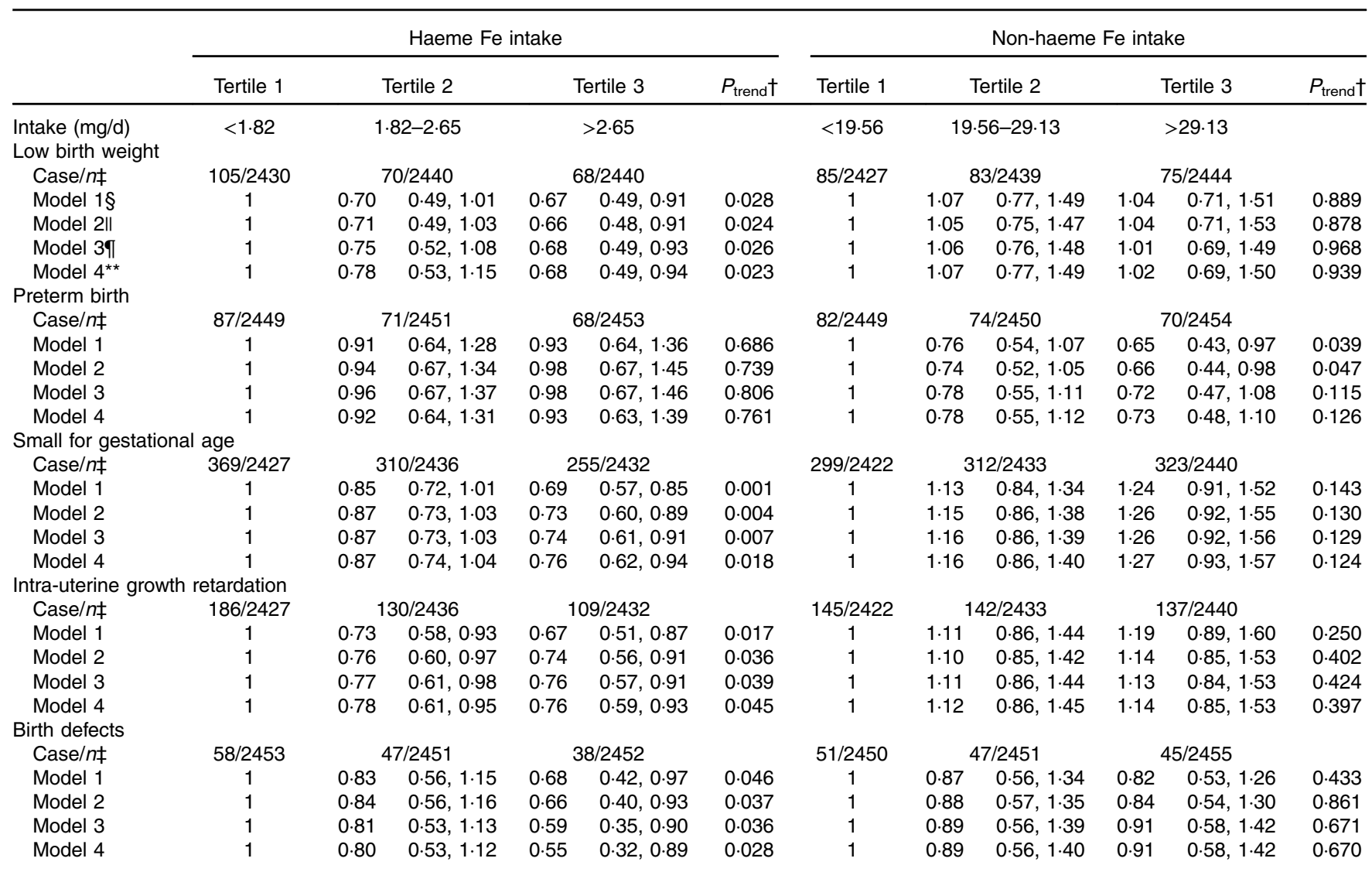

* Multilevel logistic regression models were used to estimate OR and $95 \% \mathrm{Cl}$. Models were additionally adjusted for Fe supplements use and non-haeme Fe to examine the effect of haeme $\mathrm{Fe}$, and additionally adjusted for Fe supplements use and haeme $\mathrm{Fe}$ to examine the effect of non-haeme $\mathrm{Fe}$.

$\dagger P_{\text {for trend }}$ across tertiles was calculated using the median intake of each tertile as a continuous variable.

$\ddagger$ Number of participants who had the corresponding adverse birth outcomes in the corresponding groups/the total number of participants in the corresponding groups.

$\S$ Model 1 was adjusted for energy.

॥ Model 2 was adjusted for energy and socio-demographic characteristics, including geographic area, residence, childbearing age, education, occupation, household wealth index and parity.

I Model 3 was adjusted for all variables in model 2 plus health-related characteristics, including passive smoking, alcohol drinking, antenatal check visit frequency, folate supplements use, anaemia and medication use.

** Model 4 was adjusted for all variables in model 3 plus principal component score based on the nutrient intakes.

in the lowest tertile. Pregnant women in the medium and highest tertiles of haeme Fe intake were less likely to deliver an IUGR baby relative to those in the lowest tertile (medium tertile $v$. lowest tertile: OR 0.78; $95 \%$ CI 0.61, 0.95; highest tertile $v$. lowest tertile: OR 0.76; $95 \%$ CI $0.59,0.93 ; P_{\text {trend }}=0.045$ ). We found a $45 \%$ lower risk of birth defects for the highest compared with the lowest tertile of haeme Fe intake (OR 0.55; 95\% CI 0.32, 0.89). Moreover, the risks for LBW, SGA, IUGR and birth defects were reduced with increasing tertiles of haeme Fe intake in all models $\left(P_{\text {trend }}<0 \cdot 05\right)$. However, we observed no relationship between haeme Fe intake and PTB risk.

We further explored the magnitude of change in birth weight in association with maternal haeme $\mathrm{Fe}$ intake during pregnancy (Table 4). In the energy-adjusted model, per $1 \mathrm{mg} / \mathrm{d}$ increase of haeme Fe intake resulted in an increase of $24 \mathrm{~g}$ of birth weight (95\% CI 10, 38; $P=0 \cdot 001$ ). The significant association persisted when additional adjustments were performed. After adjustment for all possible confounders (socio-demographical, health-related and dietary factors), the birth weight increase was $16 \mathrm{~g}$ (95\% CI 3, 31; $P=0.035$ ) for per $1 \mathrm{mg} / \mathrm{d}$ increase in maternal haeme Fe intake during pregnancy.

\section{Associations between iron supplements use and birth outcomes}

The association between Fe supplements use and LBW risk is shown in Fig. 2. Women taking Fe supplements during pregnancy had a lower risk of delivering a LBW baby (OR 0.72; $95 \%$ CI $0.50,0.95)$. Fe supplements use during the second and third trimesters was associated with a significantly reduced risk of LBW (during the second trimester: OR 0.67; 95\% CI 0.42 , 0.98; during the third trimester: OR 0.47 ; $95 \%$ CI $0.24,0.93$ ), whereas no association was observed between Fe supplements use during the first trimester and LBW risk. The associations of 
Fe supplements use during pregnancy or during three different gestational periods with PTB, SGA, IUGR or birth defects were not statistically significant (data not shown).

\section{Roles of modifiable factors}

The associations between maternal $\mathrm{Fe}$ intake (total $\mathrm{Fe}$ intake from diet and supplements, dietary total $\mathrm{Fe}$, haeme $\mathrm{Fe}$,

Table 4. Birth weight changes associated with per $1 \mathrm{mg} / \mathrm{d}$ increase in maternal haeme iron intake during pregnancy*

(Changes and $95 \%$ confidence intervals)

\begin{tabular}{lccc}
\hline & Change $(\mathrm{g})$ & $95 \% \mathrm{Cl}$ & $P$ \\
\hline Model 1† & 24 & 10,38 & 0.001 \\
Model 2 $\ddagger$ & 21 & 7,35 & 0.004 \\
Model 3§ & 20 & 6,35 & 0.006 \\
Model 4II & 16 & 3,31 & 0.035 \\
\hline
\end{tabular}

* Multilevel linear regression models were used to estimate changes and $95 \% \mathrm{Cl}$. Models were additionally adjusted for Fe supplements use and non-haeme Fe to examine the effect of haeme Fe.

$\dagger$ Model 1 was adjusted for energy.

‡ Model 2 was adjusted for energy and socio-demographic characteristics, including geographic area, residence, childbearing age, education, occupation, household wealth index and parity.

$\S$ Model 3 was adjusted for all variables in model 2 plus health-related characteristics, including passive smoking, alcohol drinking, antenatal check visit frequency, folate supplements use, anaemia and medication use.

॥ Model 4 was adjusted for all variables in model 3 plus principal component score based on the nutrient intakes. non-haeme Fe and Fe supplements use) and birth outcomes did not vary by vitamin $\mathrm{C}$ intake, passive smoking, alcohol drinking or anaemia (data not shown), and the tests for interaction were not significant $(P>0.05)$.

\section{Discussion}

In this population-based cross-sectional study, we observed significant and negative associations between maternal haeme Fe intake during pregnancy and birth outcomes, including LBW, SGA, IUGR and birth defects. Fe supplements use during pregnancy and during the second and third trimesters was associated with a reduced risk of LBW. We found no significant associations of total Fe from diet and supplements, dietary total Fe or non-haeme Fe intake with birth outcomes.

Previous studies on maternal Fe intake and birth outcomes have generally reported on dietary total $\mathrm{Fe}$ intake and $\mathrm{Fe}$ supplements use only. Similar to the current study, several studies reported no association of dietary total Fe intake during pregnancy with birth weight ${ }^{(13,14)}$. A recent comprehensive meta-analysis showed a significantly negative association of prenatal Fe use with LBW and no associations with PTB or $\mathrm{SGA}^{(2)}$, which is also consistent with our present study. To our knowledge, only one study conducted in the UK investigated the associations of different sources and types of Fe intakes with birth weight and gestational age. The result of this previous

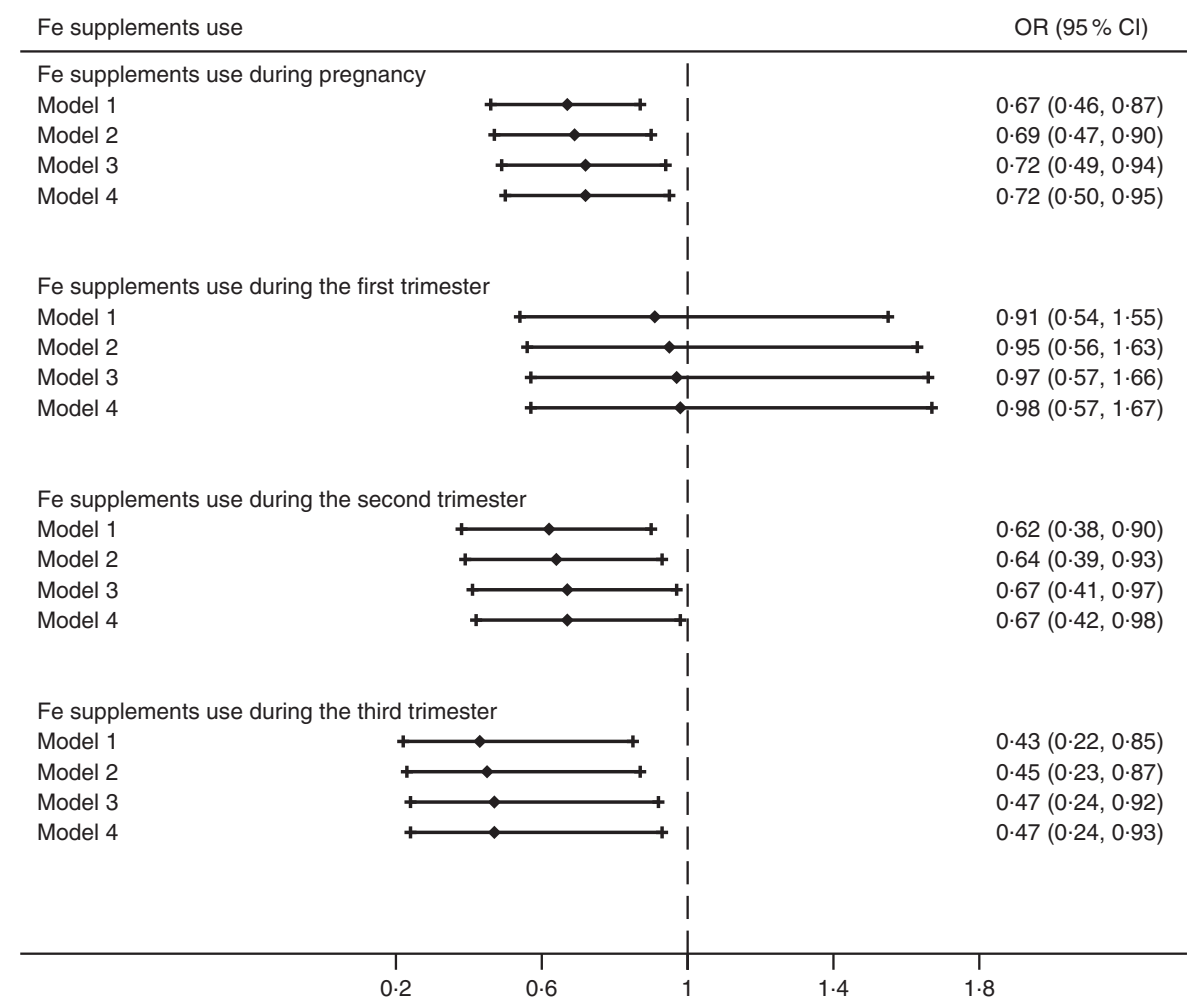

Fig. 2. OR of low birth weight associated with iron supplements use during pregnancy in Shaanxi Province, Northwest China. Multilevel logistic regression models were used to estimate OR and $95 \% \mathrm{Cl}$. Model 1 was adjusted for energy. Model 2 was adjusted for energy and socio-demographic characteristics, including geographic area, residence, childbearing age, education, occupation, household wealth index and parity. Model 3 was adjusted for all variables in model 2 plus healthrelated characteristics, including passive smoking, alcohol drinking, antenatal check visit frequency, folate supplements use, anaemia and medication use. Model 4 was adjusted for all variables in model 3 plus principal component score based on the nutrient intakes. Models were additionally adjusted for dietary total iron intake to examine the effect of iron supplements use. 
study showed a positive association between both total Fe intake and non-haeme Fe intake, derived by $24 \mathrm{~h}$ dietary recall in the first trimester, and birth weight, which is different from the result of our study. The discrepancy may be partially due to different study designs, nutrient assessment methods and population characteristics such as genetic backgrounds and dietary habits. However, the result of no association between maternal Fe intake and PTB in this previous study is in agreement with our study result. Studies on the association between maternal $\mathrm{Fe}$ intake and birth defects are sparse. A previous study reported a negative association between dietary total $\mathrm{Fe}$ intake and spinal bifida in the offspring ${ }^{(17)}$. In the current study, we observed a negative association between haeme Fe intake and birth defects. However, we cannot further evaluate the associations of maternal $\mathrm{Fe}$ intake with birth defects subtypes because of the limited cases. Further large studies with better designs are needed to explore the effect of maternal $\mathrm{Fe}$ intake on birth defects, especially for the subtypes with a high prevalence such as congenital heart defects ${ }^{(31)}$.

$\mathrm{Fe}$ is an essential constituent of hb and myoglobin, which account for $60 \%$ of total body Fe. Fe is necessary for DNA synthesis, various enzymatic processes and mitochondrial energy generation ${ }^{(1)}$, which may exert effects on pregnant women and their fetus. Dietary $\mathrm{Fe}$ is the main determinant of body Fe store, and exists as haeme and non-haeme Fe. Consistent with the current study, previous studies reported that the two forms of Fe may have different relations with health outcomes $^{(10,11)}$. Although precise mechanisms are not clear, the different bioavailability of haeme and non-haeme Fe could partially explain the discrepancy in their relations with birth outcomes. Haeme Fe absorption is more efficient and affected little by other dietary factors, whereas non-haeme Fe absorption is well regulated and depends on accompanying dietary components such as vitamin $\mathrm{C}$, the enhancer of Fe absorption, and $\mathrm{Ca}$ and polyphenols, the inhibitors of Fe absorption ${ }^{(9,32)}$. It has been reported that haeme Fe absorption was 10-15 times higher than non-haeme Fe absorption ${ }^{(10)}$. Therefore, it is plausible that higher haeme Fe intake is more likely to increase body Fe stores and thus is more likely to be associated with reduced risks for adverse birth outcomes. It is also plausible that pregnant women with a higher intake of haeme Fe may have a much better baseline Fe status because of their more concern about nutrition, such that their subsequent increase in Fe stores resulting from haeme $\mathrm{Fe}$ intake may have raised $\mathrm{Fe}$ levels enough to be beneficial. In fact, the participants in our study with a higher intake of haeme Fe tended to have better overall nutrition status, which was supported by the higher intakes of most energy-adjusted nutrients in the higher tertile of haeme Fe intake shown in Table 2. Previous studies supported significant associations between maternal $\mathrm{Fe}$ status ( $\mathrm{hb}$, ferritin and transferrin receptor) and birth outcomes (LBW, PTB and $\mathrm{SGA})^{(2,33,34)}$. Maternal hepcidin, the master regulator of systemic Fe bioavailability, was reported to influence placental uptake of dietary haeme and non-haeme $\mathrm{Fe}^{(35)}$ and relate with adverse pregnancy outcomes ${ }^{(36)}$. Moreover, a previous study demonstrated gene-Fe interaction affected birth weight ${ }^{(37)}$. People with distinct genetic backgrounds may respond variously to Fe intake and Fe status. Future studies integrating different sources and types of $\mathrm{Fe}$ intake, measures of body $\mathrm{Fe}$ status with genetic backgrounds are warranted.

The present study was conducted in Shaanxi Province of Northwest China using a stratified multistage random sampling method, with the large sample size accounting for $3 \%$ of neonates in Shaanxi, China. The Shaanxi Province was similar to the Northwest China in the diversity of culture and lifestyle. Thus, our results can be generalised to the overall Shaanxi Province and Northwest China, and may also partly reflect the status among pregnant women in China. Another strength of this study included the relatively accurate birth outcomes collected by reviewing birth certificates and medical records. However, some limitations should be acknowledged. First, the information on nutrient intakes and non-dietary characteristics during pregnancy was retrospectively self-reported by the mothers at 0-12 months (median: 3; 10th-90th percentiles: 0-7) after delivery. Although previous studies suggested that nutrient intakes and events during pregnancy could be recalled rather well even after years ${ }^{(38-40)}$, we cannot rule out the possible exposure misclassification due to recall bias. To minimise bias, we made efforts to help participants recall accurately in the study. For one thing, we used standard questionnaires and detailed supporting materials such as food portion images and calendars to gather information. For another thing, we conducted a pilot study to test the survey instruments and trained interviewers rigorously according to the detailed guides before the formal survey. Second, we cannot reveal a real causal association because of the cross-sectional design. Third, we cannot fully rule out all other unobserved and unknown confounders even after controlling for some potential confounders, including socio-demographic, health-related and dietary factors. For example, we did not collect data on maternal pre-pregnancy weight and height, which were reported to be associated with birth outcomes ${ }^{(16)}$. Fourth, we lacked data on biomarkers of body Fe status such as hb, ferritin and transferrin receptor during pregnancy, which limited us to further explore the associations between maternal Fe status and birth outcomes.

In conclusion, findings from the present study suggest that higher haeme $\mathrm{Fe}$ intake is associated with a reduced risk for LBW, SGA, IUGR and birth defects. The result also suggests that Fe supplements use during pregnancy is negatively associated with LBW risk. The increase in maternal haeme Fe intake and Fe supplements use during pregnancy may reduce adverse birth outcomes risk. Future studies with data on different sources and types of $\mathrm{Fe}$, measures of body Fe status and genetic backgrounds are warranted to further explore their associations and to decipher underlying mechanisms.

\section{Acknowledgements}

The authors are grateful to all mothers who participated in this study, all staff who coordinated field work and all investigators who contributed to data collection.

This study was supported by the National Natural Science Foundation of China (grant no.: 81230016) and the Shaanxi Health and Family Planning Commission (Sxwsjswzfcght2016-013). 
The authors' contributions are as follows: J. Y., S. D. and H. Y. conceived and designed the study; J. Y., S. D., Y. C. and H. Y. drafted and revised the manuscript; J. Y., L. P., Y. J. and F. L. analysed and interpreted the data; J. Y., L. P., L. Z., Q. W., Q. L., Y. K. and Y. S. collected and cleared the data. All authors have read and approved the final version of the manuscript.

The authors declare that there are no conflicts of interest.

\section{Supplementary material}

For supplementary material/s referred to in this article, please visit https://doi.org/10.1017/S0007114517000691

\section{References}

1. Lopez A, Cacoub P, Macdougall IC, et al. (2016) Iron deficiency anaemia. Lancet 387, 907-916.

2. Haider BA, Olofin I, Wang M, et al. (2013) Anaemia, prenatal iron use, and risk of adverse pregnancy outcomes: systematic review and meta-analysis. BMJ 346, f3443.

3. Zeng L, Dibley MJ, Cheng Y, et al. (2008) Impact of micronutrient supplementation during pregnancy on birth weight, duration of gestation, and perinatal mortality in rural western China: double blind cluster randomised controlled trial. BMJ 337, a2001.

4. Christian P, Murray-Kolb LE, Khatry SK, et al. (2010) Prenatal micronutrient supplementation and intellectual and motor function in early school-aged children in Nepal. JAMA 304, 2716-2723.

5. Peña-Rosas JP, De-Regil LM, Garcia-Casal MN, et al. (2015) Daily oral iron supplementation during pregnancy. The Cochrane Database Systematic Reviews 2015, issue 7, CD004736.

6. Li C, Zeng L, Wang D, et al. (2015) Prenatal micronutrient supplementation is not associated with intellectual development of young school-aged children. J Nutr 145, 1844-1849.

7. Andersen HS, Gambling L, Holtrop G, et al. (2006) Maternal iron deficiency identifies critical windows for growth and cardiovascular development in the rat postimplantation embryo. $J$ Nutr 136, 1171-1177.

8. Gambling L, Dunford S, Wallace DI, et al. (2003) Iron deficiency during pregnancy affects postnatal blood pressure in the rat. J Physiol 552, 603-610.

9. Hurrell R \& Egli I (2010) Iron bioavailability and dietary reference values. Am J Clin Nutr 91, 1461s-1467s.

10. Khambalia AZ, Aimone A, Nagubandi P, et al. (2015) High maternal iron status, dietary iron intake and iron supplement use in pregnancy and risk of gestational diabetes mellitus: a prospective study and systematic review. Diabet Med 33, 1211-1221.

11. Bao W, Rong Y, Rong S, et al. (2012) Dietary iron intake, body iron stores, and the risk of type 2 diabetes: a systematic review and meta-analysis. BMC Med 10, 119.

12. Lee SE, Talegawkar SA, Merialdi M, et al. (2013) Dietary intakes of women during pregnancy in low- and middle-income countries. Public Health Nutr 16, 1340-1353.

13. Mathews F, Yudkin P \& Neil A (1999) Influence of maternal nutrition on outcome of pregnancy: prospective cohort study. BMJ 319, 339-343.

14. Lagiou P, Mucci L, Tamimi R, et al. (2005) Micronutrient intake during pregnancy in relation to birth size. Eur J Nutr 44, 52-59.

15. Baker PN, Wheeler SJ, Sanders TA, et al. (2009) A prospective study of micronutrient status in adolescent pregnancy. $A m \mathrm{~J}$ Clin Nutr 89, 1114-1124.
16. Alwan NA, Greenwood DC, Simpson NA, et al. (2011) Dietary iron intake during early pregnancy and birth outcomes in a cohort of British women. Hum Reprod 26, 911-919.

17. Groenen PM, van Rooij IA, Peer PG, et al. (2004) Low maternal dietary intakes of iron, magnesium, and niacin are associated with spina bifida in the offspring. J Nutr $\mathbf{1 3 4}$, $1516-1522$

18. Shaw GM, Carmichael SL, Yang W, et al. (2010) Periconceptional nutrient intakes and risks of conotruncal heart defects. Birth Defects Res A Clin Mol Teratol 88, 144-151.

19. Zhai FY, Du SF, Wang ZH, et al. (2014) Dynamics of the Chinese diet and the role of urbanicity, 1991-2011. Obes Rev 15, Suppl. 1, 16-26.

20. Yang J, Dang S, Cheng Y, et al. (2017) Dietary intakes and dietary patterns among pregnant women in Northwest China. Public Health Nutr 20, 282-293.

21. Crozier SR, Robinson SM, Godfrey KM, et al. (2009) Women's dietary patterns change little from before to during pregnancy. J Nutr 139, 1956-1963.

22. Rifas-Shiman SL, Rich-Edwards JW, Willett WC, et al. (2006) Changes in dietary intake from the first to the second trimester of pregnancy. Paediatr Perinat Epidemiol 20, 35-42.

23. Cheng Y, Yan H, Dibley MJ, et al. (2008) Validity and reproducibility of a semi-quantitative food frequency questionnaire for use among pregnant women in rural China. Asia Pac J Clin Nutr 17, 166-177.

24. Institute of Nutrition and Food Safety \& China Center for Disease Control and Prevention (2009) China Food Composition Book 1, 2nd ed. Beijing: Peking University Medical Press.

25. Institute of Nutrition and Food Safety \& China Center for Disease Control and Prevention (2005) China Food Composition Book, 2nd ed. Beijing: Peking University Medical Press.

26. Chinese Nutrition Society (2014) Chinese Dietary Reference Intakes 2013. Beijing: Science Press.

27. Zhu L, Zhang R, Zhang S, et al. (2015) Chinese neonatal birth weight curve for different gestational age. Zhonghua $\mathrm{Er} \mathrm{Ke} \mathrm{Za}$ Zhi 53, 97-103.

28. Willett W (2013) Nutritional Epidemiology, 3rd ed. New York: Oxford University Press.

29. Filmer D \& Pritchett LH (2001) Estimating wealth effects without expenditure data - or tears: an application to educational enrollments in states of India. Demography $\mathbf{3 8}$, 115-132.

30. Schulze MB, Hoffmann K, Kroke A, et al. (2003) An approach to construct simplified measures of dietary patterns from exploratory factor analysis. Br J Nutr 89, 409-419.

31. Yang J, Qiu H, Qu P, et al. (2015) Prenatal alcohol exposure and congenital heart defects: a meta-analysis. PLOS ONE 10, $\mathrm{e} 0130681$.

32. Cook JD (1990) Adaptation in iron metabolism. Am J Clin Nutr 51, 301-308.

33. Rahman MM, Abe SK, Rahman MS, et al. (2016) Maternal anemia and risk of adverse birth and health outcomes in low- and middle-income countries: systematic review and meta-analysis. Am J Clin Nutr 103, 495-504.

34. Alwan NA, Cade JE, McArdle HJ, et al. (2015) Maternal iron status in early pregnancy and birth outcomes: insights from the Baby's Vascular health and Iron in Pregnancy study. Br J Nutr 113, 1985-1992.

35. Young MF, Griffin I, Pressman E, et al. (2012) Maternal hepcidin is associated with placental transfer of iron derived from dietary heme and nonheme sources. J Nutr 142, 33-39.

36. Simavli S, Derbent AU, Keskin EA, et al. (2015) Do the first, second and third trimester maternal serum hepcidin 
concentrations clarify obstetric complications? I Matern Fetal Neonatal Med 28, 854-857.

37. Hur J, Kim H, Ha EH, et al. (2013) Birth weight of Korean infants is affected by the interaction of maternal iron intake and GSTM1 polymorphism. J Nutr 143, 67-73.

38. Bunin GR, Gyllstrom ME, Brown JE, et al. (2001) Recall of diet during a past pregnancy. Am J Epidemiol 154, $1136-1142$
39. Bosco JL, Tseng M, Spector LG, et al. (2010) Reproducibility of reported nutrient intake and supplement use during a past pregnancy: a report from the Children's Oncology Group. Paediatr Perinat Epidemiol 24, 93-101.

40. Kvalvik LG, Nilsen RM, Skjaerven R, et al. (2012) Self-reported smoking status and plasma cotinine concentrations among pregnant women in the Norwegian Mother and Child Cohort Study. Pediatr Res 72, 101-107. 